\begin{tabular}{|c|l|}
\hline Title & New potentials for string axion inflation \\
\hline Author(s) & Kobay ashi, Tatsuo; Oikawa, A kane; Otsuka, Hajime \\
\hline Citation & $\begin{array}{l}\text { Physical review D, 93(8), 083508 } \\
\text { https://doi.org/10.1103/PhysRevD.93.083508 }\end{array}$ \\
\hline Issue Date & 2016-0414 \\
\hline Doc URL & http://hdl.handle.net/2115/61880 \\
\hline Type & article \\
\hline File Information & PhysRevD.93.083508.pdf \\
\hline
\end{tabular}

Instructions for use 
PHYSICAL REVIEW D 93, 083508 (2016)

\title{
New potentials for string axion inflation
}

\author{
Tatsuo Kobayashi, ${ }^{1, *}$ Akane Oikawa, ${ }^{2, \dagger}$ and Hajime Otsuka ${ }^{2, *}$ \\ ${ }^{1}$ Department of Physics, Hokkaido University, Sapporo 060-0810, Japan \\ ${ }^{2}$ Department of Physics, Waseda University, Tokyo 169-8555, Japan
}

(Received 2 November 2015; published 13 April 2016)

\begin{abstract}
We propose a new type of axion inflation with complex structure moduli in the framework of type IIB superstring theory compactified on the Calabi-Yau manifold. The inflaton is identified as the axion for the complex structure moduli whose potential is originating from instantonic corrections appearing through the period vector of the mirror Calabi-Yau manifold. The axionic shift symmetry is broken down to the discrete one by the inclusion of the instantonic correction and certain three-form fluxes. Our proposed inflation scenario is compatible with Kähler moduli stabilization. We also study a typical reheating temperature in the case of complex structure moduli inflation.
\end{abstract}

DOI: 10.1103/PhysRevD.93.083508

\section{INTRODUCTION}

The cosmic inflation is an attractive scenario which is tied to not only a thermal history of the Universe, but also the particle phenomenology. It is quite important to discuss the particle phenomenology and cosmology on the same footing. In particular, superstring theory is a good candidate for the unified theory of gravitational and gauge interactions, and gives us a tool to calculate the higher-dimensional operators which are sensitive to the fundamental scale.

In higher-dimensional theory as well as superstring theory, the moduli fields associated with the metric, vector, and tensor fields appear in its low-energy effective field theory. Since the scalar potential for these moduli fields are prohibited by the Lorentz and gauge symmetries, they are usually considered as candidates of inflaton fields. Moreover, in the type IIB superstring theory on the Calabi-Yau (CY) manifold, the author of Ref. [1] pointed out that the complex structure moduli and/or dilaton fields are generically stabilized by the flux-induced potential at the tree level, whereas the Kähler moduli can be stabilized at their minimum by certain nonperturbative effects. Thus, one of the Kähler moduli can play a role of the inflaton field. (See, for review, e.g., Ref. [2].) The imaginary part of the Kähler moduli and scalar fields associated with the higher-dimensional fields, i.e., the axions are also considered as candidates of the inflaton fields whose scalar potential is the form of natural inflation [3] and/or axion-monodromy inflation [4,5]. However, the complex structure moduli are not so discussed in the light of cosmic inflation and the thermal history of the Universe after the inflation. From the inflationary point of view, there are several studies in terms of complex structure moduli, for example, the natural inflation is derived from the

\footnotetext{
*kobayashi@particle.sci.hokudai.ac.jp

a.oikawa@aoni.waseda.jp

"hajime.13.gologo@akane.waseda.jp
}

nonperturbative effects through the threshold corrections $[6]^{1}$ and instanton effects [8]. The backreaction from the Kähler moduli is also discussed in Refs. [9,10]. As one of the difficulties to consider one of the complex structure moduli as the inflaton, one can expect that the Kähler moduli could be destabilized during and after the inflation because there appears a runaway direction in their potential.

In order to overcome them, we begin with the fourdimensional (4D) $\mathcal{N}=1$ supergravity derived from the type IIB string theory on the CY manifold. These instantonic effects for the prepotential of the CY manifold have been explicitly calculated by employing the technique of mirror symmetry within the framework of topological string $[11,12]$. Therefore, the flux-induced superpotential, i.e., Gukov-VafaWitten (GVW) superpotential [13], receives the world sheet instanton effects. If the relevant complex structure moduli only appear from the nonperturbative effects through the period vector of the CY manifold, their energy scale can be smaller than that of other complex structure moduli and dilaton as well as the Kähler moduli. Then, these light complex structure moduli are stabilized at their minimum after the Kähler moduli stabilization. Thus, one can discuss the scenario where the inflaton is identified with one of the complex structure moduli and its scalar potential is originating from the world sheet instanton effects in mirror CY. Unlike the result of Ref. [8], we focus on not only the class of natural inflation, but also the more general class of largefield inflation. We will show that we can derive a new type of inflation potential, which are in a sense a mixture of polynomial functions and sinusoidal functions including the natural inflation potential, and our new inflation models are favored by the recent results of Planck [14].

The remaining paper is organized as follows. In Sec. II, we review the details of quantum-corrected Kähler

\footnotetext{
${ }^{1}$ Through threshold corrections, one can realize a large decay constant enhanced by the inverse of loop factor. See also, Ref. [7].
} 
potential and GVW superpotential. Based on the moduli stabilization setup as discussed in Ref. [8], we show new types of axion inflations and their prediction of cosmological observables in Sec. III. The obtained results are favored by the recent results of Planck data and the reheating process is different from that of a usual Kähler moduli inflation. Finally, Sec. IV is our conclusion.

\section{QUANTUM-CORRECTED PERIOD VECTOR IN TYPE IIB STRING THEORY}

Here and hereafter, we adopt the reduced Planck unit, $M_{\mathrm{Pl}}=2.4 \times 10^{18} \mathrm{GeV}=1$. In type IIB superstring theory on the CYorientifold, all the complex structure moduli $(U)$ and dilaton $(S)$ can be generically stabilized by the flux-induced superpotential [1], the so-called GVW superpotential [13],

$$
W_{\mathrm{GVW}}(S, U)=\int G_{3}(S) \wedge \Omega(U),
$$

where $\Omega$ denotes the holomorphic three-form of the $\mathrm{CY}$ manifold and $G_{3}=F_{3}-i \mathrm{SH}_{3}$ is the linear combination of Ramond-Ramond $\left(F_{3}\right)$ and Neveu-Schwarz three-forms $\left(\mathrm{H}_{3}\right)$, respectively. Since these three-form fluxes along these cycles are quantized on the cycle of the CY manifold, GVW superpotential reduces to

$$
W_{\mathrm{GVW}}(S, U)=\sum_{\alpha=1}^{2 h_{-}^{1,2}+2}\left(N_{F}-i S N_{H}\right)^{\alpha} \Pi_{\alpha}
$$

where $N_{F}^{\alpha}, N_{H}^{\alpha}$ are integers and $\alpha$ run from 1 to $2 h_{-}^{1,2}+2$ with $h_{-}^{1,2}$ being the number of complex structure moduli, i.e., the hodge number of the $\mathrm{CY}$ manifold. Here, $\Pi_{\alpha}$ denote the period vector.

In the language of $4 \mathrm{D} \mathcal{N}=1$ supergravity, the Kähler potential is described by

$$
K=K(U, \bar{U})-\ln (S+\bar{S})+K(T, \bar{T}),
$$

where $T$ stand for the Kähler moduli. Then, the scalar potential is expressed by the Kähler potential (3) and the superpotential (2),

$$
V=e^{K}\left(K^{I \bar{J}} D_{I} W D_{\bar{J}} \bar{W}-3|W|^{2}\right),
$$

where $K^{I \bar{J}}$ is the inverse of the Kähler metric $K_{I \bar{J}}=$ $\partial^{2} K / \partial \Phi^{I} \partial \bar{\Phi}^{\bar{J}}$ for $\Phi^{I}=S, T^{a}, U^{i}$ with $a$ being the number of Kähler moduli, $D_{I} W=W_{I}+K_{I} W$ is the Kähler covariant derivative for $W$ with $W_{I}=\partial W / \partial \Phi^{I}$, and $K_{I}=\partial K / \partial \Phi^{I}$. From the no-scale structure for the Kähler moduli, $K^{a \bar{b}} K_{a} K_{\bar{b}}=3$, the scalar potential reduces to

$$
V=e^{K}\left(\sum_{S, U^{i}} K^{I \bar{J}} D_{I} W D_{\bar{J}} \bar{W}\right),
$$

from which the complex structure moduli and axion dilaton are stabilized at the supersymmetric minimum, $\left\langle D_{I} W\right\rangle=0$ with $\Phi^{I}=S, U^{i}$. On the other hand, the Kähler moduli would be stabilized by the perturbative string loop and $\alpha^{\prime}$ corrections [15] and/or nonperturbative corrections represented by the Kachru-Kallosh-Linde-Trivedi (KKLT) scenario [16] or large volume scenario (LVS) [17].

Although the above situation is the usual considered setup, one can also consider that some of the complex structure moduli would be stabilized through not the tree and loop interactions in the GVW superpotential, but the world sheet instanton effects involved in the GVW superpotential. In such a case, these light complex structure moduli would be stabilized at their minimum after the Kähler moduli stabilization. It implies that the scale of nonperturbative effects to stabilize the Kähler moduli is larger than that of world sheet instanton effects for the period vector. Then, one can extract the scalar potential for the light complex structure moduli whose real or imaginary parts play a role of inflaton as shown in the next section.

Such quantum corrections for the period vector have been calculated by employing the technique of mirror symmetry and $\mathcal{N}=2$ special geometry in the framework of topological string and the gauged linear sigma model on the world sheet. (See, for a review, e.g., Ref. [18].) The quantum-corrected Kähler potential for the complex structure moduli is given by $^{2}$

$$
\begin{aligned}
e^{-K(U, \bar{U})} & =i \Pi^{\dagger} \cdot \Sigma \cdot \Pi, \\
& =i\left|X^{0}\right|^{2}\left(2(F-\bar{F})-\left(U^{i}-\bar{U}^{i}\right)\left(F_{i}+\bar{F}_{i}\right)\right),
\end{aligned}
$$

where $\Sigma$ is the symplectic matrix,

$$
\Sigma=\left(\begin{array}{cc}
0 & 1 \\
-1 & 0
\end{array}\right) .
$$

The prepotential $F$ and its derivative with respect to $X^{\zeta}$ with $\zeta=0, i$ are $[20]$

$$
\begin{aligned}
F= & -\frac{1}{3 !} \kappa_{i j k} U^{i} U^{j} U^{k}-\frac{1}{2} \kappa_{i j} U^{i} U^{j}+\kappa_{i} U^{i}+\frac{1}{2} \kappa_{0} \\
& -\frac{1}{(2 \pi i)^{3}} \sum_{\beta} n_{\beta} \operatorname{Li}_{3}\left(q^{\beta}\right), \\
\mathcal{F}= & \left(X^{0}\right)^{2} F, \quad \mathcal{F}_{\zeta}=\frac{\partial}{\partial X^{\zeta}} \mathcal{F}, \quad F_{i}=\frac{\partial}{\partial U^{i}} F,
\end{aligned}
$$

where $\operatorname{Li}_{s}(z)=\sum_{n=1} \frac{z^{n}}{n^{s}}$ is the polylogarithm function and the integers $n_{\beta}$ are the genus zero Gromov-Witten invariants(world sheet instanton) labeled by $\beta$, i.e., $\beta=d_{i} \beta_{i}$ with $d_{i}$ and $\beta_{i}$ being the integers and the elements in cohomology $H_{2}\left(\tilde{M}_{\mathrm{CY}}, \boldsymbol{Z}\right) \backslash\{0\}$ of the mirror CY manifold $\tilde{M}_{\mathrm{CY}}$, respectively. The explicit form of the period vector $\Pi=$ $\left(X^{0}, X^{i}, \mathcal{F}_{0}, \mathcal{F}_{i}\right)^{T}$ is given by

\footnotetext{
${ }^{2}$ See for the case of Calabi-Yau fourfolds [19].
} 


$$
\begin{aligned}
\Pi & =X^{0}\left(\begin{array}{c}
1 \\
U^{i} \\
2 F-U^{i} \partial_{i} F \\
\partial_{i} F
\end{array}\right) \\
& =X^{0}\left(\begin{array}{c}
1 \\
\frac{1}{3 !} \kappa_{i j k} U^{i} U^{j} U^{k}+\kappa_{i} U^{i}+\kappa_{0}-\sum_{\beta} n_{\beta}^{0}\left(\frac{2}{(2 \pi i)^{3}} \operatorname{Li}_{3}\left(q^{\beta}\right)-\frac{d_{i}}{(2 \pi i)^{2}} U^{i} \operatorname{Li}_{2}\left(q^{\beta}\right)\right) \\
-\frac{1}{2} \kappa_{i j k} U^{j} U^{k}-\kappa_{i j} U^{j}+\kappa_{i}-\frac{1}{(2 \pi i)^{2}} \sum_{\beta} n_{\beta}^{0} d_{i} \operatorname{Li}_{2}\left(q^{\beta}\right)
\end{array}\right),
\end{aligned}
$$

where $\kappa_{i j k}$ is the intersection number of $\tilde{M}_{\mathrm{CY}}, q^{\beta_{i}}=e^{2 \pi i d_{i} U^{i}}$ and

$$
\begin{aligned}
\kappa_{i j k} & =\int_{\tilde{M}_{\mathrm{CY}}} J_{i} \wedge J_{j} \wedge J_{k}, \quad \kappa_{i j}=-\frac{1}{2} \int_{\tilde{M}_{\mathrm{CY}}} J_{i} \wedge J_{j} \wedge J_{j}, \\
\kappa_{j} & =\frac{1}{43 !} \int_{\tilde{M}_{\mathrm{CY}}} c_{2}\left(\tilde{M}_{\mathrm{CY}}\right) \wedge J_{j}, \quad \kappa_{0}=\frac{\zeta(3)}{(2 \pi i)^{3}} \int_{\tilde{M}_{\mathrm{CY}}} c_{3}\left(\tilde{M}_{\mathrm{CY}}\right)=\frac{\zeta(3)}{(2 \pi i)^{3}} \chi\left(\tilde{M}_{\mathrm{CY}}\right) .
\end{aligned}
$$

Here, $\chi\left(\tilde{M}_{\mathrm{CY}}\right)$ is the Euler characteristic and $\zeta(3) \simeq 1.2$.

By substituting Eqs. (7), (8), and (9) to (6), the quantum-corrected Kähler potential is brought into the following form:

$$
\begin{aligned}
e^{-K(U, \bar{U})}= & \frac{i}{6} \sum_{i j k} \kappa_{i j k}\left(U^{i}-\bar{U}^{i}\right)\left(U^{j}-\bar{U}^{j}\right)\left(U^{k}-\bar{U}^{k}\right)-\frac{\zeta(3)}{4 \pi^{3}} \chi\left(\tilde{M}_{\mathrm{CY}}\right)+\frac{i}{(2 \pi i)^{2}} \sum_{\beta} d_{i} n_{\beta}\left(U^{i}-\bar{U}^{i}\right)\left(\mathrm{Li}_{2}\left(q^{\beta}\right)+\operatorname{Li}_{2}\left(\bar{q}^{\beta}\right)\right) \\
& -\frac{2 i}{(2 \pi i)^{3}} \sum_{\beta} n_{\beta}\left(\operatorname{Li}_{3}\left(q^{\beta}\right)+\operatorname{Li}_{3}\left(\bar{q}^{\beta}\right)\right)
\end{aligned}
$$

where $X^{0}$ is chosen as unity. The term proportional to the Euler characteristic is the $\left(\alpha^{\prime}\right)^{3}$ correction descended from the $\mathcal{R}^{4}$ term in the ten-dimensional action [21]. The third and fourth terms in Eq. (11) denote the world sheet instanton effects which break the continuous shift symmetry to the discrete one.

In the following, we change the normalization for the complex structure moduli $U^{i}$ as $i U^{i}$ and correspondingly the Kähler potential is rewritten as

$$
\begin{aligned}
e^{-K(U, \bar{U})}= & \frac{1}{6} \sum_{i j k} \kappa_{i j k}\left(U^{i}+\bar{U}^{i}\right)\left(U^{j}+\bar{U}^{j}\right)\left(U^{k}+\bar{U}^{k}\right)-\frac{\zeta(3)}{4 \pi^{3}} \chi\left(\tilde{M}_{\mathrm{CY}}\right) \\
& +\frac{2}{(2 \pi)^{2}} \sum_{\beta} \sum_{n=1}^{\infty} d_{i} n_{\beta}\left(U^{i}+\bar{U}^{i}\right) \frac{1}{n^{2}} \cos \left(-i \pi n \sum_{j} d_{j}\left(U^{j}-\bar{U}^{j}\right)\right) e^{-\pi n \sum_{k} d_{k}\left(U^{k}+\bar{U}^{k}\right)} \\
& +\frac{4}{(2 \pi)^{3}} \sum_{\beta} \sum_{n=1}^{\infty} n_{\beta} \frac{1}{n^{3}} \cos \left(-i \pi n \sum_{j} d_{j}\left(U^{j}-\bar{U}^{j}\right)\right) e^{-\pi n \sum_{k} d_{k}\left(U^{k}+\bar{U}^{k}\right)}
\end{aligned}
$$

\section{NEW TYPE OF AXION INFLATION}

In this section, we show the illustrative models where one of the axions of complex structure moduli plays a role of inflaton. The inflation potential is a mixture of polynomial functions and sinusoidal functions, which are descended from the instantonic effects for the period vector of the CY manifold. We assume that the Kähler moduli are also stabilized at the KKLT (LVS) minimum in Secs. III A 1 and III A 2, respectively.

\section{A. Moduli stabilization}

In the following, we denote the $(n-2)$ complex structure moduli and axion dilaton by $z$, whereas the other complex structure moduli are represented as $U_{1}$ and $U_{2}$. By 
choosing certain three-form fluxes (2) in the large complex structure limit $\left(|z|,\left|U_{1}\right|,\left|U_{2}\right| \gg 1\right)$, their Kähler potential and superpotential are extracted as

$$
\begin{aligned}
K(\operatorname{Re} S, \operatorname{Re} U) & =-\ln \left[f_{0}\left(\operatorname{Re} z, \operatorname{Re} U_{1}, \operatorname{Re} U_{2}\right)\right], \\
W(S, U) & =g_{0}(z)+g_{1}(z)\left(U_{2}+N U_{1}\right),
\end{aligned}
$$

where $f_{0}$ denotes the first line of Eq. (12) determined by the topological number of the CY manifold, whereas $g_{0,1}(z)$ depends on the quanta of three-form fluxes and $N$ is the integer. Note that the GVW superpotential is a fourth-order polynomial function at the tree level. In analogy of Ref. [8], when we redefine the complex structure moduli as

$$
\begin{aligned}
& \Psi \equiv U_{2}+N U_{1}, \\
& \Phi \equiv U_{2},
\end{aligned}
$$

the Kähler potential and superpotential are also rewritten as

$$
\begin{aligned}
K(\operatorname{Re} S, \operatorname{Re} U) & =-\ln \left[f_{0}(\operatorname{Re} z,(\operatorname{Re} \Psi-\operatorname{Re} \Phi) / N, \operatorname{Re} \Phi)\right], \\
W(S, U) & =g_{0}(z)+g_{1}(z) \Psi
\end{aligned}
$$

which implies that $\Psi$ and $z$ would be stabilized at the supersymmetric minimum satisfying $D_{I} W=0$ with $I=\Psi$, $z$. Correspondingly, the real part of $\Phi$ can be stabilized at the supersymmetric minimum at $K_{\Phi}=0$, where its mass is given by the nonvanishing superpotential. Then, the imaginary part of $\Phi$ remains massless at this stage and $\operatorname{Im} \Phi$ becomes the inflaton whose potential is generated by the instanton effects.

Before going to the detail of instanton effects for $\operatorname{Im} \Phi$, we comment on the moduli stabilization for the Kähler moduli. So far, $\operatorname{Re} \Phi, z, \Psi$ fields are stabilized at the perturbative level. When the nonperturbative effects for the Kähler moduli appear in the superpotential, the Kähler moduli can be stabilized at the scale above the energy scale of the remaining complex structure modulus $\operatorname{Im} \Phi$. As shown later, the mechanism to stabilize the Kähler moduli is irrelevant to the dynamics of the inflation whose inflaton corresponds to one of the complex structure moduli, if all the Kähler moduli are stabilized at the scale above the energy scale of remaining complex structure moduli $\Phi$. Thus, one can consider the KKLT scenario or LVS for the Kähler moduli stabilization with some uplifting scenario. In the case of the KKLT scenario, $w=\langle W(S, U)\rangle$ is sufficiently small in order to be compatible with the scale of nonperturbative effects for the Kähler moduli, whereas $w$ is of order unity in the LVS.

In a certain CY manifold, the instanton effects are explicitly calculated by employing the mirror symmetry. In the large complex structure moduli limit, one can neglect the exponential term in the Kähler potential and superpotential due to the large-field values of complex structure moduli $z, \Phi, \Psi$. However, if we include these corrections, they are represented as

$$
\begin{aligned}
\Delta K \simeq & -\frac{1}{\left\langle f_{0}\right\rangle} \sum_{i=1}^{2} f_{1}^{(i)}\left(\frac{2}{\pi}+\left(U_{i}+\bar{U}_{i}\right)\right) \\
& \times \cos \left(-i \pi\left(U_{i}-\bar{U}_{i}\right)\right) e^{-\pi\left(U_{i}+\bar{U}_{i}\right)}, \\
\Delta W \simeq & \sum_{i=1}^{2}\left(g_{2}^{(i)}+g_{3}^{(i)} U_{i}\right) e^{-2 \pi U_{i}},
\end{aligned}
$$

where we choose the integer $d_{i}=1$ and the heavy modulidependent parameters $g_{2,3}^{(i)}, f_{1}^{(i)}$ are now taken as real, for simplicity. The corrections for the Kähler potential are extracted in the large complex structure limit. Along the line of Ref. [8], we omit the exponential term for $U_{2}$ under

$$
e^{-2 \pi\left\langle\operatorname{Re} U_{2}\right\rangle} \ll e^{-2 \pi\left\langle\operatorname{Re} U_{1}\right\rangle} \ll 1
$$

Then, the Kähler potential and superpotential reduce to

$$
\begin{aligned}
\Delta K \simeq & -\frac{f_{1}^{(1)}}{\left\langle f_{0}\right\rangle}\left(\frac{2}{\pi}+\frac{\Psi+\bar{\Psi}-\Phi-\bar{\Phi}}{N}\right) \\
& \times \cos \left(-i \pi \frac{\Psi-\bar{\Psi}-\Phi+\bar{\Phi}}{N}\right) e^{-\pi \frac{\Psi+\bar{\Psi}-\Phi-\bar{\Phi}}{N}}, \\
\Delta W \simeq & \left(g_{2}^{(1)}+\frac{g_{3}^{(1)}}{N}(\Psi-\Phi)\right) e^{-2 \pi \frac{\Psi-\Phi}{N}},
\end{aligned}
$$

on the basis $(\Psi, \Phi)$.

We assume that the other complex structure moduli are enough heavier than the imaginary part of $\Phi$ and they are fixed at their supersymmetric minimum. Furthermore, the Kähler moduli are also fixed at their minimum during and after the inflation. This assumption is ensured as follows. In our setup, the inflation occurs due to the nonvanishing $\Delta W$ which is mainly determined by the real parts of $(\langle\Psi\rangle,\langle\Phi\rangle)$. Thus, one can choose $\Delta W$ to be smaller than the tree-level part of GVW superpotential $w=\langle W\rangle$ and nonperturbative superpotential for the Kähler moduli. In addition, we assume that the nonperturbative effects relevant for the Kähler moduli have to be independent to the remaining light complex structure modulus $\Phi$, otherwise one have to consider the stabilization of Kähler moduli and $\Phi$, simultaneously. After the stabilization of heavy complex structure moduli and axion dilaton, it is then found that the superpotential behaves effectively constant $w$ and Kähler moduli can be stabilized at the KKLT or LVS without depending on the dynamics of the lightest complex structure modulus, as far as the Kähler moduli are enough heavier than the lightest modulus. 


\section{Inflaton potential based on KKLT scenario}

First of all, we assume that the Kähler moduli are stabilized at the supersymmetric minimum satisfying $D_{T} W=0$ based on the KKLT scenario, where the superpotential is effectively given by $W=w+W_{\text {non }}(\langle T\rangle) \equiv w_{0}$ with $W_{\text {non }}(T)$ being certain nonperturbative effects such as gaugino condensation [22] and D-brane instanton effects. ${ }^{3}$ It is then further assumed that the prefactor of nonperturbative effects for the Kähler moduli do not depend on $\Phi$. In such a case, when the gauginos living on $N$ stacks of
D7-branes condensate, the nonperturbative superpotential $W_{\text {non }}(T)$ is described as $W_{\text {non }}(T) \simeq e^{-a T}$ with $a=8 \pi^{2} / N$. The mass scales of Kähler moduli, $m_{T}^{2} \simeq\left(a m_{3 / 2}\right)^{2}$ with $m_{3 / 2} \simeq e^{(K(\operatorname{Re} S, \operatorname{Re} U)+K(T, \bar{T})) / 2} w$, are much heavier than the remaining complex structure modulus which is determined by $\Delta W$.

Next, we now turn to the dynamics of the lightest complex structure modulus $\operatorname{Im} \Phi$. With the tree-level and quantumcorrected Kähler potential and superpotential (15), (18), the superpotential and its covariant derivative become

$$
\begin{aligned}
W \simeq & w_{0}+\Delta W \\
D_{\Phi} W \simeq & \Delta W_{\Phi}+\Delta K_{\Phi} W \\
\simeq & e^{-2 \pi\left\langle\operatorname{Re} U_{1}\right\rangle}\left[e^{-2 \pi i \frac{\operatorname{Im} \Psi\rangle-\operatorname{Im} \Phi}{N}}\left(-\frac{g_{3}^{(1)}}{N}+\frac{2 \pi}{N}\left(g_{2}^{(1)}+\frac{g_{3}^{(1)}}{N}(\langle\Psi\rangle-\Phi)\right)\right)\right. \\
& \left.-\frac{w_{0} f_{1}^{(1)}}{\left\langle f_{0}\right\rangle}\left(-\frac{1}{N} \cos \left(2 \pi \frac{\langle\operatorname{Im} \Psi\rangle-\operatorname{Im} \Phi}{N}\right)+\frac{\pi}{N}\left(\frac{2}{\pi}+2\left\langle\operatorname{Re} U_{1}\right\rangle\right) e^{-2 \pi i \frac{(\operatorname{Im} \Psi\rangle-\operatorname{Im} \Phi}{N}}\right)\right],
\end{aligned}
$$

in the limit of $w_{0} \gg \Delta W$. These lead to the scalar potential for $\tilde{\phi}=\operatorname{Im} \Phi,{ }^{4}$

$$
\begin{aligned}
e^{-K-\Delta K} V \simeq & K^{\Phi \bar{\Phi}} D_{\Phi} W D_{\bar{\Phi}} \bar{W}-3|W|^{2} \\
\simeq & -3|W|^{2}+K^{\Phi \bar{\Phi}} e^{-4 \pi\left\langle\operatorname{Re} U_{1}\right\rangle}\left[A^{2}+B^{2}(\langle\operatorname{Im} \Psi\rangle-\tilde{\phi})^{2}\right. \\
& \left.+\left(C^{2}+2 A C\right) \cos ^{2}\left(2 \pi \frac{\langle\operatorname{Im} \Psi\rangle-\tilde{\phi}}{N}\right)+B C(\langle\operatorname{Im} \Psi\rangle-\tilde{\phi}) \sin \left(4 \pi \frac{\langle\operatorname{Im} \Psi\rangle-\tilde{\phi}}{N}\right)\right] \\
\simeq & -3 w_{0}^{2}-6 w_{0} e^{-2 \pi\left\langle\operatorname{Re} U_{1}\right\rangle}\left[\left(g_{2}^{(1)}+\frac{g_{3}^{(1)}}{N}\left\langle\operatorname{Re} U_{1}\right\rangle\right) \cos \left(2 \pi \frac{\langle\operatorname{Im} \Psi\rangle-\tilde{\phi}}{N}\right)\right. \\
& \left.+\frac{g_{3}^{(1)}}{N}(\langle\operatorname{Im} \Psi\rangle-\tilde{\phi}) \sin \left(2 \pi \frac{\langle\operatorname{Im} \Psi\rangle-\tilde{\phi}}{N}\right)\right] \\
& -3 e^{-4 \pi\left\langle\operatorname{Re} U_{1}\right\rangle}\left[\left(g_{2}^{(1)}+\frac{g_{3}^{(1)}}{N}\left\langle\operatorname{Re} U_{1}\right\rangle\right)^{2}+\left(\frac{g_{3}^{(1)}}{N}\right)^{2}(\langle\operatorname{Im} \Psi\rangle-\tilde{\phi})^{2}\right] \\
& +K^{\Phi \bar{\Phi}} e^{-4 \pi\left\langle\operatorname{Re} U_{1}\right\rangle}\left[A^{2}+B^{2}(\langle\operatorname{Im} \Psi\rangle-\tilde{\phi})^{2}\right. \\
& \left.+\left(C^{2}+2 A C\right) \cos ^{2}\left(2 \pi \frac{\langle\operatorname{Im} \Psi\rangle-\tilde{\phi}}{N}\right)+B C(\langle\operatorname{Im} \Psi\rangle-\tilde{\phi}) \sin \left(4 \pi \frac{\langle\operatorname{Im} \Psi\rangle-\tilde{\phi}}{N}\right)\right],
\end{aligned}
$$

where $K=K(\operatorname{Re} S, \operatorname{Re} U)+\Delta K+K(T, \bar{T})$ is the full Kähler potential of moduli fields. The other parameters are defined as

$$
\begin{aligned}
& A=-\frac{g_{3}^{(1)}}{N}+\frac{2 \pi g_{2}^{(1)}}{N}+\frac{2 \pi g_{3}^{(1)}}{N}\left\langle\operatorname{Re} U_{1}\right\rangle-\frac{w_{0} f_{1}^{(1)} 2 \pi}{\left\langle f_{0}\right\rangle N}\left(\frac{1}{\pi}+\left\langle\operatorname{Re} U_{1}\right\rangle\right), \\
& B=\frac{2 \pi g_{3}^{(1)}}{N^{2}}, \quad C=\frac{w_{0} f_{1}^{(1)}}{\left\langle f_{0}\right\rangle N} .
\end{aligned}
$$

\footnotetext{
${ }^{3}$ The backreaction from the Kähler moduli is left for future work.

${ }^{4}$ In the framework of LVS, the $-3|W|^{2}$-term in the scalar potential is canceled by the extended no-scale structure of Kähler moduli [23] as shown in Sec. III A 2.
} 
When the complex structure moduli are canonically normalized at the Minkowski minimum by setting certain uplifting such as the anti-D-brane [16] and F-term uplifting scenario [24,25], the inflaton potential is approximately given in the large complex structure limit $\left\langle\operatorname{Re} U_{1}\right\rangle \gg 1$,

$$
V_{\mathrm{inf}} \simeq \Lambda_{1}\left(1-\cos \frac{\phi}{M_{1}}\right)+\Lambda_{2} \phi \sin \frac{\phi}{M_{1}},
$$

where $\phi \equiv k_{1}(\langle\operatorname{Im} \Psi\rangle-\tilde{\phi})$ with $k_{1}$ being the normalization factor of $\mathcal{O}\left(\left\langle\operatorname{Re} U_{2}\right\rangle\right) ; \quad M_{1}=N k_{1} / 2 \pi$ and $\Lambda_{1,2} \simeq$ $\mathcal{O}\left(e^{\langle K\rangle} w_{0} e^{-2 \pi\left\langle\operatorname{Re} U_{1}\right\rangle}\right)$ are the real constants. The first term is the same as the potential form of natural inflation, but the second term as well as this full potential is new. Note that although this potential form looks slightly similar to the natural inflation with modulation, ${ }^{5}$ the second term is not a small correction, but two terms are comparable with each other.

So far, we have neglected the higher order terms of the world sheet instanton effects, because the constant $w_{0}$ dominates the superpotential. When the Kähler moduli are stabilized at the minimum suggested by Kallosh-Linde [29], the constant $w_{0}$ can be taken as $w_{0} \sim 0$ due to the cancelation between the nonperturbative effects for the Kähler moduli $W_{\text {non }}(\langle T\rangle)$ and the part of the tree-level GVW superpotential $w$. The mass scales of Kähler moduli, $m_{T}^{2} \sim w^{2}$, are also much heavier than that of the remaining complex structure modulus which is determined by the higher order terms of the world sheet instanton effects. In such a case, the inflaton potential is dominated by the order of $e^{-4 \pi\left\langle\operatorname{Re} U_{1}\right\rangle}$ in the scalar potential (20),

$$
V_{\mathrm{inf}} \simeq \Lambda_{3} \phi^{2},
$$

where $\phi \equiv k_{2}(\langle\operatorname{Im} \Psi\rangle-\tilde{\phi})$ with $k_{2}$ being the normalization factor of $\mathcal{O}\left(\left\langle\operatorname{Re} U_{2}\right\rangle\right)$ and $\Lambda_{3} \simeq \mathcal{O}\left(e^{\langle K\rangle} e^{-4 \pi\left\langle\operatorname{Re} U_{1}\right\rangle}\right)$ are constants by setting a specific uplifting sector in the same way as Eq. (22). However, in this situation, we need to tune the parameter in the sector of Kähler moduli so that the mass scale of $\operatorname{Re} \Phi$ is larger than that of inflaton. The obtained scalar potential is just that of chaotic inflation.

\section{Inflaton potential based on the large volume scenario}

Next, we assume that the Kähler moduli are stabilized at the nonsupersymmetric minimum within the framework of LVS, where the $\alpha^{\prime}$ corrections and nonperturbative effects are included in the Kähler potential and superpotential, respectively.

In the same way as the case of Sec. III A 1, we consider that the Kähler moduli are enough heavier than the remaining complex structure modulus $\operatorname{Im} \Phi$. Because of the extended no-scale structure of the Kähler moduli [23],

\footnotetext{
${ }^{5}$ See, e.g., Refs. [26-28].
}

the $-3|W|^{2}$-term in the scalar potential is vanishing and Eq. (20) reduces to

$$
\begin{aligned}
V \simeq & e^{K+\Delta K} K^{\Phi \bar{\Phi}} D_{\Phi} W D_{\bar{\Phi}} \bar{W} \\
\simeq & e^{K+\Delta K} K^{\Phi \bar{\Phi}} e^{-4 \pi\left\langle\operatorname{Re} U_{1}\right\rangle}\left[A^{2}+B^{2}(\operatorname{Im} \Psi-\tilde{\phi})^{2}\right. \\
& +\left(C^{2}+2 A C\right) \cos ^{2}\left(2 \pi \frac{\langle\operatorname{Im} \Psi\rangle-\tilde{\phi}}{N}\right) \\
& \left.+B C(\langle\operatorname{Im} \Psi\rangle-\tilde{\phi}) \sin \left(4 \pi \frac{\langle\operatorname{Im} \Psi\rangle-\tilde{\phi}}{N}\right)\right] .
\end{aligned}
$$

This leads to the following scalar potential for the canonically normalized field $\phi \equiv k_{3}(\langle\operatorname{Im} \Psi\rangle-\tilde{\phi})$ with $k_{3}$ being the normalization factor of $\mathcal{O}\left(\left\langle\operatorname{Re} U_{2}\right\rangle\right)$ :

$V_{\mathrm{inf}} \simeq \Lambda_{4} \phi^{2}+\Lambda_{5} \phi \sin \left(\frac{\phi}{M_{3}}\right)+\Lambda_{6}\left(1-\cos \left(\frac{\phi}{M_{3}}\right)\right)$,

where $M_{3}=N k_{3} / 4 \pi, \Lambda_{4,5,6} \simeq \mathcal{O}\left(e^{\langle K\rangle} e^{-4 \pi\left\langle\operatorname{Re} U_{1}\right\rangle}\right)$ are constants. Here we set some uplifting sector to obtain the Minkowski minimum. Thus, this potential is also new and a mixture of polynomial functions and sinusoidal functions. These three terms are comparable with each other.

Although we have neglected a backreaction from the Kähler moduli, its scalar potential is roughly estimated as

$$
V_{\mathrm{LVS}} \sim e^{K(S, U)} \frac{|W|^{2}}{\mathcal{V}^{3}},
$$

where $\mathcal{V}$ denotes the volume of the CY manifold. From Eq. (26), the scalar potential for $\phi$ appearing from Eq. (26) is of order $\mathcal{O}\left(e^{\langle K(S, U)\rangle} w_{0} e^{-2 \pi\left\langle\operatorname{Re} U_{1}\right\rangle} / \mathcal{V}^{3}\right)$. Thus, under

$$
e^{\langle K(S, U)\rangle} \frac{w_{0} e^{-2 \pi\left\langle\operatorname{Re} U_{1}\right\rangle}}{\mathcal{V}^{3}}<e^{\langle K(S, U)\rangle} \frac{e^{-4 \pi\left\langle\operatorname{Re} U_{1}\right\rangle}}{\mathcal{V}^{2}},
$$

the potential arising from the backreaction of Kähler moduli can be negligible. This situation can be realized with the mild large volume of the CY manifold, e.g., $\mathcal{V} \simeq 10^{2}, \operatorname{Re} U_{1} \simeq 0.7$, and $w_{0} \simeq 1$. When we include the backreaction from the Kähler moduli, it induces the scalar potential as in Eq. (22). Then, the total scalar potential is described by that of Eq. (25) with $\Lambda_{5,6}>\Lambda_{4}$. We leave the detailed study of the backreaction to future work. Note that the energy scale of Kähler moduli $V_{\text {LVS }}$ should be also larger than the inflation scale $V_{\text {inf }}$, i.e., $V_{\text {inf }} \ll V_{\mathrm{LVS}}$.

\section{B. Numerical analyses}

In this section, we study the inflaton dynamics characterized by three types of inflaton potential, (22), (23), and (25). First of all, the inflaton potential in Eq. (23) is just the 
form of chaotic inflation which is disfavored by the recent result of Planck. In addition to it, the inflaton potential (22) is that of natural inflation with the sinusoidal term. In our setup, as discussed in Ref. [8], the axion decay constant takes the value of the trans-Planckian due to the nature of winding trajectory on the $(\Psi, \Phi)$ plane, i.e., $M_{2} \gg 1$. Thus, the scalar potential of natural inflation without the sinusoidal term (22) is agreement with the Planck data [14] under its large axion decay constant,

$$
\begin{aligned}
P_{\xi} & =2.20 \pm 0.10 \times 10^{-9}, \\
n_{s} & =0.9655 \pm 0.0062, \\
r & <0.11,
\end{aligned}
$$

where $P_{\xi}$ is the power spectrum of the curvature perturbation and $r$ denotes the tensor-to-scalar ratio at the pivot scale $k_{*}=0.05 \mathrm{Mpc}^{-1}$. In what follows, we choose that the inflation scale is set to realize the correct magnitude of the power spectrum of curvature perturbation. For the inflaton potential (22), the slow-roll parameters are estimated as

$$
\begin{aligned}
\epsilon & =\frac{1}{2}\left(\frac{\partial_{\phi} V}{V}\right)^{2}=\frac{1}{2}\left(\frac{\left(\frac{\Lambda_{1}}{M_{1}}+\Lambda_{2}\right) \sin \frac{\phi}{M_{1}}+\Lambda_{2} \frac{\phi}{M_{1}} \cos \frac{\phi}{M_{1}}}{\left.\Lambda_{1}-\cos \frac{\phi}{M_{1}}\right)+\Lambda_{2} \phi \sin \frac{\phi}{M_{1}}}\right)^{2} \\
& \simeq \frac{2}{\phi^{2}}-\frac{1}{3 M_{1}^{2}} \frac{\Lambda_{1}+4 M_{1} \Lambda_{2}}{\Lambda_{1}+2 M_{1} \Lambda_{2}} \\
\eta & =\frac{\partial_{\phi} \partial_{\phi} V}{V}=\frac{\frac{1}{M_{1}}\left(\frac{\Lambda_{1}}{M_{1}}+2 \Lambda_{2}\right) \cos \frac{\phi}{M_{1}}-\Lambda_{2} \frac{\phi}{M_{1}^{2}} \sin \frac{\phi}{M_{1}}}{\Lambda_{1}\left(1-\cos \frac{\phi}{M_{1}}\right)+\Lambda_{2} \phi \sin \frac{\phi}{M_{1}}} \\
& \simeq \frac{2}{\phi^{2}}-\frac{5}{6 M_{1}^{2}} \frac{\Lambda_{1}+4 M_{1} \Lambda_{2}}{\Lambda_{1}+2 M_{1} \Lambda_{2}}
\end{aligned}
$$

$$
\begin{gathered}
n_{s}=1-6 \epsilon+2 \eta \simeq 1-\frac{8}{\phi^{2}}+\frac{1}{3 M_{1}^{2}}\left(1+\frac{2 M_{1} \Lambda_{2}}{\Lambda_{1}+2 M_{1} \Lambda_{2}}\right), \\
r=16 \epsilon \simeq \frac{32}{\phi^{2}}-\frac{16}{3 M_{1}^{2}}\left(1+\frac{2 M_{1} \Lambda_{2}}{\Lambda_{1}+2 M_{1} \Lambda_{2}}\right) .
\end{gathered}
$$

It is found that $n_{s}(r)$ tends to be small (large) when $\Lambda_{2}$ becomes negative. We now take into account the condition that the inflaton mass should be positive at least at a vacuum, that is, $\partial^{2} V / \partial \phi^{2}=\left(\Lambda_{1}+2 M_{1} \Lambda_{2}\right) / M_{1}^{2}>0$. These features are also captured in Fig. 1. However, as can be seen in Fig. 1, there are no sizable differences between the prediction of $n_{s}$ and $r$ of natural inflation with and without the sinusoidal term. In any case, the large axion decay constant is favored by the Planck data [14] in the light of the spectral tilt of curvature perturbation. The typical values of cosmological observables associated with a sufficient $e$-folding number are summarized in Table I.

Finally, let us discuss the scalar potential in Eq. (25). The slow-roll parameters are also estimated as

$$
\begin{aligned}
\epsilon & =\frac{1}{2}\left(\frac{2 \Lambda_{4} \phi+\left(\Lambda_{5}+\frac{\Lambda_{6}}{M_{3}}\right) \sin \frac{\phi}{M_{3}}+\frac{\Lambda_{5}}{M_{3}} \phi \cos \frac{\phi}{M_{3}}}{\phi^{2}+\Lambda_{5} \phi \sin \frac{\phi}{M_{3}}+\Lambda_{6}\left(1-\cos \frac{\phi}{M_{3}}\right)}\right)^{2} \\
& \simeq \frac{2}{\phi^{2}}-\frac{4 M_{3} \Lambda_{5}+\Lambda_{6}}{3 M_{3}^{2}\left(2 M_{3}\left(M_{3} \Lambda_{4}+\Lambda_{5}\right)+\Lambda_{6}\right)}+\mathcal{O}\left(\frac{1}{M_{3}^{4}}\right), \\
\eta & =\frac{2 \Lambda_{4}-\frac{\Lambda_{5}}{M_{3}^{2}} \phi \sin \frac{\phi}{M_{3}}+\frac{1}{M_{3}}\left(2 \Lambda_{5}+\frac{\Lambda_{6}}{M_{3}}\right) \cos \frac{\phi}{M_{3}}}{\Lambda_{4} \phi^{2}+\Lambda_{5} \phi \sin \frac{\phi}{M_{3}}+\Lambda_{6}\left(1-\cos \frac{\phi}{M_{3}}\right)} \\
& \simeq \frac{2}{\phi^{2}}-\frac{5\left(4 M_{3} \Lambda_{5}+\Lambda_{6}\right)}{6 M_{3}^{2}\left(2 M_{3}\left(M_{3} \Lambda_{4}+\Lambda_{5}\right)+\Lambda_{6}\right)}+\mathcal{O}\left(\frac{1}{M_{3}^{4}}\right),
\end{aligned}
$$

and the $n_{s}$ and $r$ become

which leads to the expressions of $n_{s}$ and $r$,

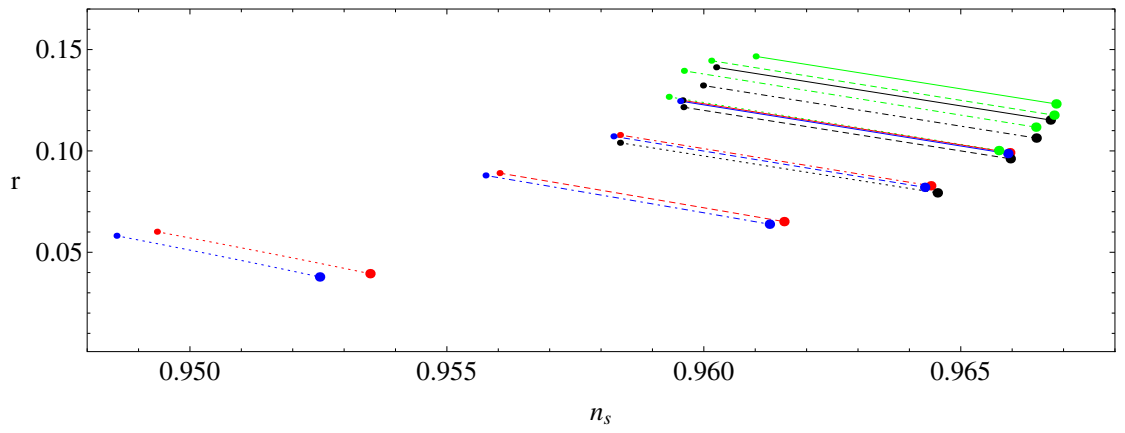

FIG. 1. Predictions of $\left(n_{s}, r\right)$ in the range of $e$-folding number, $50 \leq N_{e} \leq 60$ for the inflaton potential (22) with and without sinusoidal term. The leftmost (rightmost) circle on each line represents for the $e$-folding number $N_{e}=50\left(N_{e}=60\right)$. The black line corresponds to the prediction of natural inflation. For the red, blue and green lines, the sinusoidal term is included as $\Lambda_{2} / \Lambda_{1}=1,5$, $-1 / 6 M_{1}$, respectively. The solid, dot-dashed, dashed, and dotted lines denote the axion decay constants $M_{1}=15,12,10,8$, respectively. 
TABLE I. The input parameters $M_{1}, \Lambda_{2} / \Lambda_{1}$ and the output values of the $e$-folding number $N_{e}$, spectral tilt of curvature perturbation $n_{s}$, tensor-to-scalar ratio $r$ and the running of spectral index $d n_{s} / d \ln k$.

\begin{tabular}{lccccc}
\hline \hline$M_{1}$ & $\Lambda_{2} / \Lambda_{1}$ & $N_{e}$ & $n_{s}$ & \multicolumn{1}{c}{$r$} & $d n_{s} / d \ln k$ \\
\hline 8 & 5 & 50 & 0.96 & 0.04 & -0.0005 \\
8 & 5 & 60 & 0.964 & 0.03 & -0.0003 \\
10 & 5 & 50 & 0.964 & 0.055 & -0.0006 \\
10 & 5 & 60 & 0.969 & 0.041 & -0.0004 \\
12 & 5 & 50 & 0.966 & 0.063 & -0.0006 \\
12 & 5 & 60 & 0.971 & 0.049 & -0.0004 \\
15 & 5 & 50 & 0.968 & 0.07 & -0.0006 \\
15 & 5 & 60 & 0.973 & 0.056 & -0.0004 \\
\hline \hline
\end{tabular}

$$
\begin{aligned}
n_{s} & \simeq 1-\frac{8}{\phi^{2}}+\frac{4 M_{3} \Lambda_{5}+\Lambda_{6}}{3 M_{3}^{2}\left(2 M_{3}\left(M_{3} \Lambda_{4}+\Lambda_{5}\right)+\Lambda_{6}\right)}+\mathcal{O}\left(\frac{1}{M_{3}^{4}}\right), \\
r & \simeq \frac{32}{\phi^{2}}-\frac{16\left(4 M_{3} \Lambda_{5}+\Lambda_{6}\right)}{3 M_{3}^{2}\left(2 M_{3}\left(M_{3} \Lambda_{4}+\Lambda_{5}\right)+\Lambda_{6}\right)}+\mathcal{O}\left(\frac{1}{M_{3}^{4}}\right) .
\end{aligned}
$$

Then, it is found that $n_{s}(r)$ tends to be large (small) when $\Lambda_{5}$ becomes large compared with $\Lambda_{4}$ in the case of $M_{3} \gg 1$. We now take into account the condition that the inflaton mass should be positive at least at a vacuum, that is, $\partial^{2} V / \partial \phi^{2}=\left(2 M_{3}\left(M_{3} \Lambda_{4}+\Lambda_{5}\right)+\Lambda_{6}\right) / M_{3}>0 . \quad$ These features are also captured in Fig. 2. With suitable parameters, the cosmological observables associated with a sufficient $e$-folding number are summarized in Table II which are within the central value of recent Planck data [14]. From Fig. 2, it is found that the small- and large-field inflations can be realized without changing the spectral tilt of adiabatic curvature perturbation. The small-field inflation is achieved by the plateau appearing in the scalar potential.

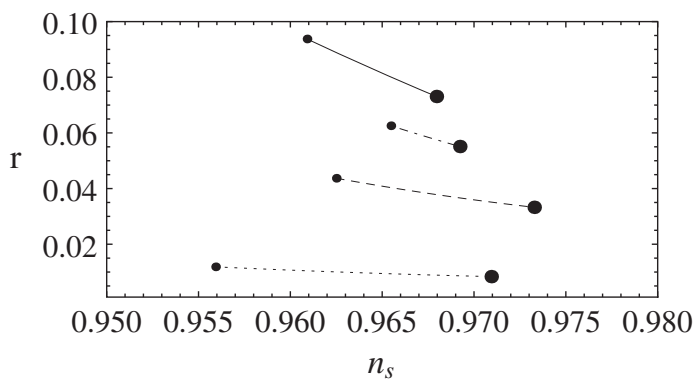

FIG. 2. Predictions of $\left(n_{s}, r\right)$ in the range of $e$-folding number, $50 \leq N_{e} \leq 60$ for the inflaton potential (25) with $\Lambda_{4} / \Lambda_{6}=1$ and $\Lambda_{5} / \Lambda_{6}=5$. The leftmost (rightmost) circle on each line represents for the $e$-folding number $N_{e}=50\left(N_{e}=60\right)$. The black solid, black dot-dashed, black dashed, and black dotted lines correspond to the axion decay constants $M_{3}=6,5,4,3$, respectively.
TABLE II. The input parameters $M_{3}, \Lambda_{4} / \Lambda_{6}, \Lambda_{5} / \Lambda_{6}$ and the output values of the $e$-folding number $N_{e}$, spectral tilt of curvature perturbation $n_{s}$, tensor-to-scalar ratio $r$, and the running of spectral index $d n_{s} / d \ln k$.

\begin{tabular}{lcccccr}
\hline \hline$M_{3}$ & $\Lambda_{4} / \Lambda_{6}$ & $\Lambda_{5} / \Lambda_{6}$ & $N_{e}$ & \multicolumn{1}{c}{$n_{s}$} & $r$ & $d n_{s} / d \ln k$ \\
\hline 5 & 1 & 5 & 60 & 0.969 & 0.05 & -0.0007 \\
5 & 1 & 5 & 55 & 0.965 & 0.06 & -0.0008 \\
5 & 1 & 5 & 50 & 0.962 & 0.07 & -0.0009 \\
3 & 1 & 5 & 60 & 0.97 & 0.008 & 0.0009 \\
3 & 1 & 5 & 55 & 0.964 & 0.097 & 0.0009 \\
3 & 1 & 5 & 50 & 0.956 & 0.012 & 0.0009 \\
5 & $1 / 5$ & 1 & 60 & 0.968 & 0.05 & -0.0007 \\
5 & $1 / 5$ & 1 & 55 & 0.965 & 0.06 & -0.0008 \\
\hline \hline
\end{tabular}

\section{Reheating temperature}

In the previous numerical analyses, we roughly estimate the amount of $e$-folding within the range $50 \leq N_{e} \leq 60$. However, it is sensitive to the thermal history of the Universe after the inflation. Indeed, the reheating temperature gives the significant effect as can be seen from the simple formula of the amount of $e$-folding [30]

$$
N_{e} \simeq 62+\ln \frac{V_{*}^{1 / 4}}{10^{16} \mathrm{GeV}}+\ln \frac{V_{*}^{1 / 4}}{V_{\text {end }}^{1 / 4}}-\frac{1}{3} \ln \frac{V_{\text {end }}^{1 / 4}}{\rho_{R}^{1 / 4}},
$$

where $V_{*}^{1 / 4}\left(V_{\text {end }}^{1 / 4}\right)$ is the energy density of scalar potential at the horizon exit (inflation end), and $\rho_{R}^{1 / 4}=\left(\pi^{2} g_{*} / 30\right) T_{R}$ is the energy density of radiation, and $g_{*}$ is the effective degrees of freedom of the radiation at the reheating temperature $T_{R}$.

In this section, we show the typical reheating temperature when one of the complex structure moduli plays a role of inflaton in three types of inflaton potential (22)-(25). After the inflation, the energy of inflaton is converted into that of matter fields in the visible and hidden sectors through the inflaton decay into them. In the string inflation with Kähler moduli, the inflaton mainly decays into the gauge bosons, gauginos, or Higgs fields due to a dimensional counting. (See, for more details, e.g., Ref. [31] in the case of LVS.) However, the complex structure moduli do not appear in the gauge kinetic function at the tree level unlike the case of the Kähler moduli. In the following analysis, we assume that the supersymmetry breaking is caused by the Kähler moduli or matter fields at a scale larger than the inflation scale. Therefore, below the inflation scale, the effective theory would be described by that of the standard model. The following mass formula of Weyl fermions:

$$
m_{I J}=e^{G / 2}\left(\nabla_{I} G_{J}+\frac{1}{3} G_{I} G_{J}\right),
$$

with $\quad \nabla_{I} G_{J}=\partial G_{J} / \partial \Phi^{I}-\Gamma_{I J}^{K} G_{K}, \quad G=K+\ln (\bar{W} W)$, $G_{I}=\partial G / \partial \Phi^{I}$, and $\Gamma_{I J}^{K}$ is the Christoffel symbol 
constructed by the metric $K_{I J}$, leads to the mass of the Weyl fermion paired with the inflaton field $(\psi)$

$$
m_{\psi \psi} \simeq e^{G / 2} G_{\Phi \bar{\Phi}},
$$

which is almost of the same order as the supersymmetry breaking scale due to $G_{\Phi} \simeq 0$. When we neglect the inflaton decay into $\psi$, the leading decay channels are categorized into the following three types of them.

First of all, the inflaton decays into the gauge bosons in the standard model through the moduli-dependent one-loop corrections in the gauge kinetic function [32],

$$
\mathcal{L}=-\frac{1}{4 g_{a}^{2}} F_{\mu \nu}^{a} F^{a \mu \nu}-\frac{1}{4} \frac{\Delta(\Phi)}{16 \pi^{2}} F_{\mu \nu}^{a} F^{a \mu \nu}
$$

where $\Delta(\Phi)$ is unknown threshold correction for $\Phi^{6}$ and $a=1,2,3$ denote the gauge groups in the standard model, $U(1)_{Y}, S U(2)_{L}$, and $S U(3)_{C}$, respectively. Thus, its decay width is estimated as

$$
\begin{aligned}
\Gamma_{\phi}^{(1)} \equiv & \sum_{a=1}^{3} \Gamma\left(\phi \rightarrow g^{(a)}+g^{(a)}\right) \\
& =\sum_{a=1}^{3} \frac{N_{G}^{a}}{128 \pi}\left(\frac{\partial_{\phi}(\Delta(\Phi)) g_{a}^{2}}{16 \pi^{2} d}\right)^{2} \frac{m_{\phi}^{3}}{M_{\mathrm{Pl}}^{2}} \\
& \simeq 5.8 \times 10^{-5}\left(\frac{m_{\phi}}{10^{13} \mathrm{GeV}}\right)^{3} \mathrm{GeV}
\end{aligned}
$$

where $\sum_{a} N_{G}^{a}=12, d \simeq \mathcal{O}\left(\sqrt{K_{\Phi \bar{\Phi}}}\right) \simeq \mathcal{O}(1), m_{\phi}$ is the inflaton mass, and $\left(g_{a}\right)^{2} \simeq 0.53$ is the gauge coupling at the grand unification scale $2 \times 10^{16} \mathrm{GeV}^{7}$ We now assume that the threshold correction is provided by the linear term of $\Phi$ in the large complex structure limit, that is, $\Delta(\Phi)=\Phi$ as can be also seen in the toroidal background [33]. When the total decay width of inflaton is dominated by the above decay, the reheating temperature is roughly estimated by equaling the Hubble parameter at the reheating and the total decay width,

$$
\begin{aligned}
\Gamma_{\phi}^{(1)} & \simeq H\left(T_{R}\right), \\
\Rightarrow T_{R} & =\left(\frac{\pi^{2} g_{*}}{90}\right)^{-1 / 4} \sqrt{\Gamma_{\phi}^{(1)} M_{\mathrm{Pl}}} \\
& \simeq 6.4 \times 10^{6}\left(\frac{m_{\phi}}{10^{13} \mathrm{GeV}}\right)^{3 / 2} \mathrm{GeV},
\end{aligned}
$$

\footnotetext{
${ }^{6}$ The explicit form of it on the toroidal background can be shown in Ref. [33].

${ }^{7}$ Although we implicitly assume that the minimal supersymmetric standard model can be realized at the grand unification scale, the size of gauge coupling is not relevant to our following analysis.
}

where $g_{*}=106.75$ is the effective degrees of freedom of the radiation at the reheating in the standard model. The inflaton decay into the gaugino pairs is prohibited when the gauginos are heavier than the inflaton. Also, anomalyinduced inflaton decays are a subdominant effect because of $K_{\Phi} \simeq 0$ [34].

The next one is the inflaton decay into the matter fields $Q$ in the standard model. Their couplings would appear from the following Kähler potential ${ }^{8}$ :

$$
K \simeq f(T+\bar{T}) e^{-2 \pi \operatorname{Re} U_{1}} \cos \left(\frac{\phi}{M}\right)|Q|^{2},
$$

where $M$ is the axion decay constant of inflaton, and $f(T+\bar{T})$ is some Kähelr moduli-dependent function. Such an instantonic coupling is the leading one between the axion inflaton and matter fields. This is because the axion does not appear at the tree-level Kähler potential due to its shift symmetry and at the nonperturbative level, the continuous shift symmetry is broken into the discrete one. It is then found that the above coupling does not lead to the inflaton decay into the matter fermions or bosons, because the supersymmetry is broken at the scale larger than the inflation scale.

Finally, we explore the couplings between the inflaton and matter fields in the superpotential, although they are higher order terms. The relevant interactions would appear from the Yukawa couplings,

$$
W=Y_{i j k}(\Phi) Q^{i} Q^{j} Q^{k},
$$

where $Y_{i j k}(\Phi)$ denotes the physical Yukawa coupling between the canonically normalized matter chiral multiplets $Q_{i}$ in the visible sector (see, e.g., Ref. [35] in the case of toroidal background). That leads to the following decay width:

$\Gamma_{\phi}^{(2)} \equiv \Gamma\left(\phi \rightarrow \psi^{i} \psi^{j} Q^{k}\right) \simeq \frac{1}{64(2 \pi)^{3}}\left\langle\frac{\partial Y_{i j k}}{\partial \phi}\right\rangle^{2} \frac{m_{\phi}^{3}}{M_{\mathrm{Pl}}^{2}}$,

where $\psi^{i}$ and $Q^{i}$ denote the matter fermion and boson in the chiral multiplet $Q^{i}$, respectively. The associated reheating temperature is roughly estimated as

$$
\begin{aligned}
\Gamma_{\phi}^{(2)} & \simeq H\left(T_{R}\right), \\
\Rightarrow T_{R} & =\left(\frac{\pi^{2} g_{*}}{90}\right)^{-1 / 4} \sqrt{\Gamma_{\phi}^{(2)} M_{\mathrm{Pl}}} \\
& \simeq 8.8 \times 10^{7}\left\langle\partial_{\phi} Y_{i j k}\right\rangle\left(\frac{m_{\phi}}{10^{13} \mathrm{GeV}}\right)^{3 / 2} \mathrm{GeV},
\end{aligned}
$$

with $g_{*}=106.75$, from which the reheating temperature is determined by the first derivative of the Yukawa coupling with respect to the inflaton field.

\footnotetext{
${ }^{8} \mathrm{We}$ use the same notation between the inflaton $\phi$ and its supermultiplet.
} 
As a result, the relevant inflaton couplings are different from those of Kähler moduli and correspondingly one can distinguish the thermal history of the Universe after the inflation from that given by the Kähler moduli inflation. We will study these phenomenological aspects through concrete models elsewhere.

\section{CONCLUSION}

In this paper, we have studied the axion inflation with complex structure moduli within the framework of type IIB string theory on the Calabi-Yau manifold. In the usual moduli stabilization procedure in type IIB string theory, all of the complex structure moduli and dilaton are stabilized at the tree level in terms of three-form fluxes, whereas the Kähler moduli are stabilized at the nonperturbative level. However, along the line of Ref. [8], here we also have pointed out that the relevant complex structure moduli can be stabilized at the minimum through the instatonic correction in the period vector of mirror Calabi-Yau manifold. Then, the masses of these complex structure moduli could be lower than those of Kähler moduli. Based on this setup, we find the new type of axion inflation associated with the lightest complex structure modulus and its scalar potential is the mixture of polynomial functions and sinusoidal functions. The new type of axion inflation is favored by the recent Planck data.

The complex structure moduli inflation is different from Kähler moduli inflation in the light of the thermal history of the Universe after the inflation. Since the couplings between the complex structure moduli and the matter fields in the visible sector are different from those of Kähler moduli, the inflaton decay channel and the related reheating process are completely changed. Although the reheating process is highly model dependent, we show the typical decay channel and reheating temperature. It would be an important direction to construct the realistic phenomenological model combined with the complex structure moduli inflation. That will be studied elsewhere.

\section{ACKNOWLEDGMENTS}

The authors thank H. Abe for useful discussions. T. K. was supported in part by the Grant-in-Aid for Scientific Research No. 25400252 and No. 26247042 from the MEXT in Japan. H. O. was supported in part by a Grant-in-Aid for JSPS Fellows No. 26-7296.
[1] S. B. Giddings, S. Kachru, and J. Polchinski, Hierarchies from fluxes in string compactifications, Phys. Rev. D 66, 106006 (2002).

[2] D. Baumann and L. McAllister, Inflation and String Theory (Cambridge University Press, Cambridge, 2015).

[3] K. Freese, J. A. Frieman, and A. V. Olinto, Natural Inflation with Pseudo-Nambu-Goldstone Bosons, Phys. Rev. Lett. 65, 3233 (1990).

[4] L. McAllister, E. Silverstein, and A. Westphal, Gravity waves and linear inflation from axion monodromy, Phys. Rev. D 82, 046003 (2010).

[5] E. Silverstein and A. Westphal, Monodromy in the CMB: Gravity waves and string inflation, Phys. Rev. D 78, 106003 (2008).

[6] H. Abe, T. Kobayashi, and H. Otsuka, Natural inflation with and without modulations in type IIB string theory, J. High Energy Phys. 04 (2015) 160.

[7] H. Abe, T. Kobayashi, and H. Otsuka, Towards natural inflation from weakly coupled heterotic string theory, Prog. Theor. Exp. Phys. 2015, 63E02 (2015).

[8] A. Hebecker, P. Mangat, F. Rompineve, and L. T. Witkowski, Winding out of the swamp: Evading the weak gravity conjecture with F-term winding inflation?, Phys. Lett. B 748, 455 (2015).

[9] H. Hayashi, R. Matsuda, and T. Watari, Issues in complex structure moduli inflation, arXiv:1410.7522.

[10] A. Hebecker, P. Mangat, F. Rompineve, and L. T. Witkowski, Tuning and backreaction in F-term axion monodromy inflation, Nucl. Phys. B894, 456 (2015).
[11] E. Witten, Topological sigma models, Commun. Math. Phys. 118, 411 (1988).

[12] E. Witten, in Mirror Symmetry I, edited by S. T. Yau (American Mathematical Society, Providence, 1998), pp. 121-160.

[13] S. Gukov, C. Vafa, and E. Witten, CFT's from Calabi-Yau four folds, Nucl. Phys. B584, 69 (2000); Nucl. Phys. B608, 477 (2001).

[14] P. A. R. Ade et al. (Planck Collaboration), Planck 2015. XX. Constraints on inflation, arXiv:1502.02114.

[15] M. Berg, M. Haack, and B. Kors, On Volume Stabilization by Quantum Corrections, Phys. Rev. Lett. 96, 021601 (2006).

[16] S. Kachru, R. Kallosh, A. D. Linde, and S. P. Trivedi, de Sitter vacua in string theory, Phys. Rev. D 68, 046005 (2003).

[17] V. Balasubramanian, P. Berglund, J. P. Conlon, and F. Quevedo, Systematics of moduli stabilisation in CalabiYau flux compactifications, J. High Energy Phys. 03 (2005) 007.

[18] K. Hori and C. Vafa, Mirror symmetry, arXiv:hep-th/ 0002222.

[19] Y. Honma and M. Manabe, Exact Kahler potential for Calabi-Yau fourfolds, J. High Energy Phys. 05 (2013) 102.

[20] S. Hosono, A. Klemm, S. Theisen, and S. T. Yau, Mirror symmetry, mirror map and applications to complete intersection Calabi-Yau spaces, Nucl. Phys. B433, 501 (1995).

[21] M. B. Green and S. Sethi, Supersymmetry constraints on type IIB supergravity, Phys. Rev. D 59, 046006 (1999). 
[22] S. Ferrara, L. Girardello, and H. P. Nilles, Breakdown of local supersymmetry through gauge fermion condensates, Phys. Lett. 125B, 457 (1983).

[23] M. Cicoli, J. P. Conlon, and F. Quevedo, Systematics of string loop corrections in type IIB Calabi-Yau flux compactifications, J. High Energy Phys. 01 (2008) 052.

[24] O. Lebedev, H. P. Nilles, and M. Ratz, de Sitter vacua from matter superpotentials, Phys. Lett. B 636, 126 (2006).

[25] E. Dudas, C. Papineau, and S. Pokorski, Moduli stabilization and uplifting with dynamically generated F-terms, J. High Energy Phys. 02 (2007) 028; H. Abe, T. Higaki, T. Kobayashi, and Y. Omura, Moduli stabilization, F-term uplifting and soft supersymmetry breaking terms, Phys. Rev. D 75, 025019 (2007); R. Kallosh and A. D. Linde, O’KKLT, J. High Energy Phys. 02 (2007) 002; H. Abe, T. Higaki, and T. Kobayashi, More about F-term uplifting, Phys. Rev. D 76, 105003 (2007).

[26] T. Kobayashi and F. Takahashi, Running spectral index from inflation with modulations, J. Cosmol. Astropart. Phys. 01 (2011) 026.

[27] M. Czerny, T. Kobayashi, and F. Takahashi, Running spectral index from large-field inflation with modulations revisited, Phys. Lett. B 735, 176 (2014).

[28] T. Kobayashi, O. Seto, and Y. Yamaguchi, Axion monodromy inflation with sinusoidal corrections, Prog. Theor. Exp. Phys. 2014, 103E01 (2014); T. Higaki, T. Kobayashi,
O. Seto, and Y. Yamaguchi, Axion monodromy inflation with multi-natural modulations, J. Cosmol. Astropart. Phys. 10 (2014) 025.

[29] R. Kallosh and A. D. Linde, Landscape, the scale of SUSY breaking, and inflation, J. High Energy Phys. 12 (2004) 004.

[30] A. R. Liddle and D. H. Lyth, The cold dark matter density perturbation, Phys. Rep. 231, 1 (1993).

[31] T. Higaki, K. Kamada, and F. Takahashi, Higgs, moduli problem, baryogenesis and large volume compactifications, J. High Energy Phys. 09 (2012) 043; T. Higaki and F. Takahashi, Dark radiation and dark matter in large volume compactifications, J. High Energy Phys. 11 (2012) 125.

[32] L. J. Dixon, V. Kaplunovsky, and J. Louis, Moduli dependence of string loop corrections to gauge coupling constants, Nucl. Phys. B355, 649 (1991).

[33] D. Lust and S. Stieberger, Gauge threshold corrections in intersecting brane world models, Fortschr. Phys. 55, 427 (2007); R. Blumenhagen, B. Kors, D. Lust, and S. Stieberger, Four-dimensional string compactifications with D-branes, orientifolds and fluxes, Phys. Rep. 445, 1 (2007).

[34] M. Endo, F. Takahashi, and T. T. Yanagida, Anomalyinduced inflaton decay and gravitino-overproduction problem, Phys. Lett. B 658, 236 (2008).

[35] D. Cremades, L. E. Ibanez, and F. Marchesano, Computing Yukawa couplings from magnetized extra dimensions, J. High Energy Phys. 05 (2004) 079. 